\title{
Physics-Based and Retina-Inspired Technique for Image Enhancement
}

\author{
Mohamed Sedky, Ange A.Malek Aly and Tomasz Bosakowski \\ School of Computing, Staffordshire University, Beaconside, Stafford, United Kingdom \\ \{m.h.sedky, ange.aly, t.bosakowski\}@staffs.ac.uk
}

Keywords: Retinex, Image Enhancement, Image Formation Models, Colour Constancy, Contrast Constancy

\begin{abstract}
This paper develops a novel image/video enhancement technique that integrates a physics-based image formation model, the dichromatic model, with a retina-inspired computational model, multiscale model of adaptation. In particular, physics-based features (e.g. Power Spectral Distribution of the dominant illuminant in the scene and the Surface Spectral Reflectance of the objects contained in the image are estimated and are used as inputs to the multiscale model for adaptation. The results show that our technique can adapt itself to scene variations such as a change in illumination, scene structure, camera position and shadowing and gives superior performance over the original model.
\end{abstract}

\section{INTRODUCTION}

Image enhancement plays a fundamental role in computer vision. Images and videos with good lightness and contrast are a strong requirement in several areas of applications, where human experts make an important decision based on the imaging information, such as medical, security, forensic and remote sensing applications (Saichandana, 2014).

The objective of an image enhancement algorithm is to improve the information perception in images/videos for a human viewer and/or to support an image/video processing algorithm with a better input. The result, of such algorithms, is a processed image that is more suitable than the original image for a specific application (Maini and Aggarwal, 2010).

Typically, the enhancement is performed to improve certain desired features e.g. image contrast, lightness or grey-levels distribution. The choice of these features and the way they are modified are specific to a given task.

The well-known methods of image enhancement include spatial and frequency enhancement techniques such as linear transformation, gamma correction, contrast stretching, histogram equalisation, homomorphic filtering, etc. Those conventional methods are easy to implement but generally do not recover exact true colour of the images hence they have limited application areas.

For example, one of the drawbacks of histogram equalisation is generating colour distortion in images during enhancement.
Conventional image enhancement methods have been widely used with their different advantages and drawbacks; since the last century, there has been increased interest in retina-inspired techniques e.g. Retinex and Cellular Neural Networks (CNN), as they attempt to mimic the human retina.

Retinex, a compound word comprised of retina and cortex, is a computational theory derived from anatomy and neuroscience. Retinex is a very popular and effective method to remove environmental light interferences. The two main concepts behind the theory are: (1) colours of objects are determined by the capability to reflect rays of light, and (2) colours are not influenced by uneven illumination. The main advantages of Retinex are image sharpening and colour constancy (Land, 1986).

Despite considerable advances in computer vision techniques, the human eye and visual cortex by far supersede the performance of state-of-the-art algorithms.

Marr's approach to studying computer vision starts by following what is called representational theories of minds (Marr and Vision, 1982). He argues that in order to understand fully a particular machine carrying out a particular information-processing task, we have to study that machine as well as the information-processing task. He saw the computational study of vision as tightly linked to psychophysics and neurophysiology. But the last three decades have seen some weakening of that integration.

Georgeson and Sullivan (Georgeson and Sullivan, 1975) have studied apparent contrast at different 
spatial frequencies, in foveal and peripheral vision, under photopic and scotopic conditions, and at various orientations in astigmatic subjects. In their experiments, they found that despite extreme differences in the contrast thresholds for two patterns, they will match in apparent contrast when their physical contrasts are equal. They have concluded that in man, visual information is blurred by optical and neural processes restored by an active process of compensation; they have defined this ability as 'contrast constancy'.

Similarly, humans have the ability to separate the illumination power spectral distribution from the surface spectral reflectance when judging object appearance, 'colour constancy' (Ho et al., 1990).

Image enhancement approaches may be classified depending on the image representation used as nonphysics-based or physics-based. Non-physics based image enhancement methods use one of the known colour spaces as a cue to model the scene. While the word physics refers to the extraction of intrinsic features about the materials contained in the scene based on an understanding of the underlying physics which govern the image formation. This process is achieved by applying physics-based image formation models that attempt to estimate or eliminate the illumination and/or the geometric parameters to extract information about the surface spectral reflectance (SSR) (Sedky, 2014).

Recently, physics-based image formation models e.g. Dichromatic model, have gained the attention of the computer vision research community as they describe mathematically how a digital image is formed inside a camera, and they attempt to estimate the Power Spectral Distribution (PSD) of the dominant illuminant as well as the SSR of objects.

In this paper, we propose a novel physics-based and retina-inspired image/video enhancement technique that integrates a physics-based image formation model, the dichromatic model, with a retina-inspired computational model, a multiscale model for adaptation proposed in (Pattanaik et al., 1998). In particular, physics-based features (e.g. PSD of the dominant illuminant and the SSR of the objects contained in the image) are estimated and are used as inputs to a retina-inspired algorithm to achieve both colour and contrast constancy to overcome the limitations of conventional image/video enhancement methods.

The rest of the paper is organized as follows: the following Section gives a background on Retinainspired models as well as image formation models, Section 3 describes our computational model, Section 4 presents an illustrative visual comparison between our proposed technique and the multiscale model for adaptation, and finally Section 5 concludes the paper.

\section{BACKGROUND}

Retinex algorithms can be categorised into three classes, which are: path-based, recursive and centre/surround Retinex algorithms (Le, 2014).

A. Path-based algorithm: The original work of Land (Land and McCann, 1971) (Land, 1986) belongs to this category. In this algorithm, the lightness of each pixel depends on the multiplication of ratios along random walks. The drawbacks of this algorithm are the dependency of the path geometry and the computational complexity, which has a lot of parameters.

B. Recursive algorithm: It was developed by Frankly and McCann (Frankle and McCann, 1983). This algorithm works on long distance iterations between pixels, then moves to short distance interactions. The spacing between pixels being compared decreases at each step in clockwise order. This algorithm is computationally more efficient than the path-based one. The main drawback is that it depends on the number of times a pixel's neighbours are to be visited. This parameter is named some iterations. The optimal value for this parameter is not clear and can negatively influence the final result (Ciurea and Funt, 2004).

C. Centre/Surround algorithm (Morel, 2010): This technique introduces a weight in the reciprocal influence of two pixels (Finlayson, 1995), which is inversely proportional to their square distance.

The lightness values are computed by subtracting a blurred version of the input image from the original image. This algorithm is faster than the path-based one, with less parameter. The main drawback is the "grey world" assumption, which was addressed (Berns et al., 1993) by introducing the multi-scale Retinex with colour restoration technique (Reddy, 2013).

\subsection{Image Formation Models}

Appearances of scenes depend on four fundamental elements: an illuminant, a medium, a material and a vision system. The illuminant represents the source of visible electromagnetic energy and is characterised by its PSD. The medium is the medium in which electromagnetic waves travel. The surface of the material modulates the incident electromagnetic energy and is represented by the surface spectral reflectance, the fraction of incident radiation reflected by this surface. The vision system is identified by the spectral sensitivities of its photosensitive sensors that represent the response of such element to the received reflected light. 


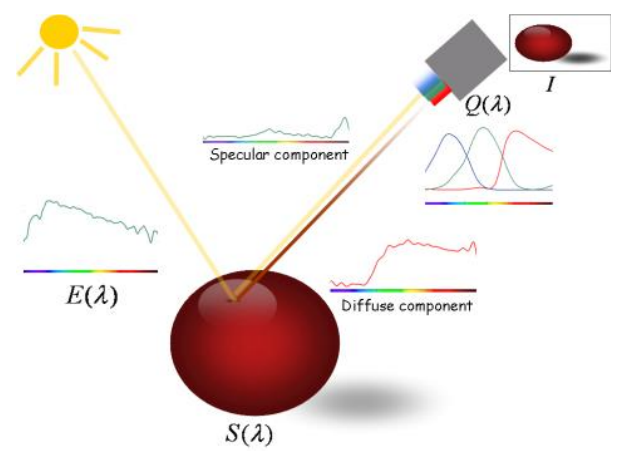

Figure 1: Schematic diagram of image formation

Another important parameter is the set of geometrical features, which represent the scene structure, the illuminant orientation, the surface roughness and the viewing geometry. These features combine non-linearly to form a digital image (Sedky, 2014).

Recovering these features from images is an important problem in image processing; however, this recovery is generally hard with the limited amount of information provided by standard commercial imaging devices.

\section{The Dichromatic Model}

The dichromatic model represents the light reflected by an inhomogeneous dielectric material as a linear combination of diffuse and specular reflections. Each of these parts is further divided into two elements, one accounting for the geometry and another purely spectral. There are a great number of reflection models, most of them developed in the field of computer graphics. Among these methods, the dichromatic reflection model is a usual choice for those algorithms employing a physical model to represent color images, as shown in Figure 1, and the equation below:

$$
I_{\mathrm{c}}=w_{\mathrm{d}} \int_{\Omega}^{E}(\lambda) S(\lambda) Q_{\mathrm{c}}(\lambda) d \lambda+w_{\mathrm{s}} \int_{\Omega}^{E}(\lambda) Q_{\mathrm{c}}(\lambda) d \lambda
$$

Where $\Omega$ is the visible range from $400 \mathrm{~nm}$ to $700 \mathrm{~nm}, I_{c}$ is the measured color intensity of the reflected light, $w_{d}$ and $w_{s}$ are geometrical parameters for diffuse and specular reflection respectively, $E(\lambda)$ is the spectral power distribution function of the illumination, $S(\lambda)$ is the SSR of the object, $Q_{c}(\lambda)$ is the camera sensor spectral sensitivities characteristic, and $c$ represents the color channels (Red, Green and Blue).

Assuming Lambertian surfaces, theoretically, an image taken by a digital colour camera (for diffuse only reflection) can be described as:

$$
I_{\mathrm{c}}=w_{\mathrm{d}} \int_{\Omega} E(\lambda) S(\lambda) Q_{\mathrm{c}}(\lambda) d \lambda
$$

\section{Surface Spectral Reflectance Estimation Linear Models}

Several researchers (Bajcsy et al., 1990), (Maloney, 1986) and (Marimont and Wandell, 1992) show that both illumination and surface spectral reflectance are relatively smooth functions of the wavelength of light in the visual spectrum and that they can be expressed using finite-dimensional linear models.

The surface reflectances of a great variety of materials have been studied. Parkkinnen et al. (Parkkinen et al., 1989), Maloney (Maloney, 1986) studied the reflectance properties of the Munsell chips, which is a database of experimentally measured surface spectral reflectance characteristics. Parkkinen concludes that 8 basis functions can cover almost all existing data in Munsell chips database. However, it has been shown in the literature (Klinker et al., 1990) that the spectral reflectance calculated using the first three basis functions has average error 0.0055 and 0.01 . Figure 2 shows the first 4 Parkkinen basis functions. As it can be seen from the figure, a significant characteristic of Parkkinen basis functions is the flat distribution of his first basis function.

$$
S(\lambda)=\sum_{i=1}^{n} w_{i} \phi_{i}(\lambda)
$$

where $\phi_{i}(\lambda)$ is the $i^{\text {th }}$ reflectance linear basis function, and $w_{i}$ is its corresponding weight, $n$ is the number of basis functions used.

Using finite-dimensional linear models to represent the surface spectral reflectance provides a compact description of data, with few basis functions we can represent surface spectral reflectance for general materials. The linear models have been extensively used in some colour constancy algorithms where the main aim was to recover either the illumination or the reflectance functions, or both of them.

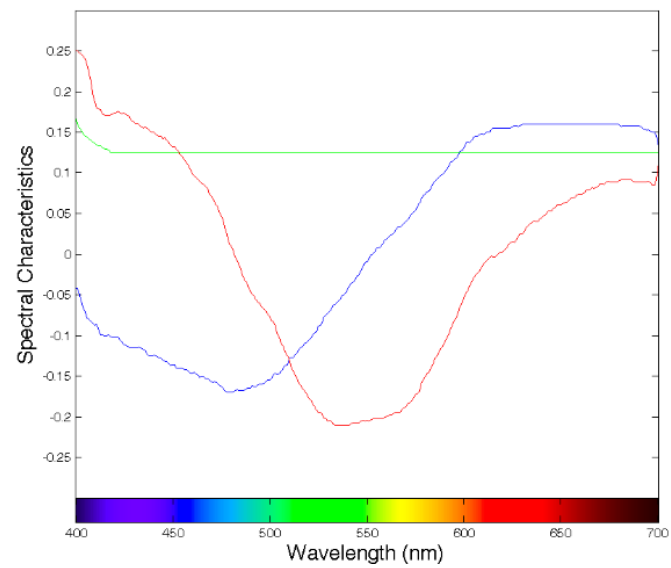

Figure 2: Parkkinen's first three basis functions 
The assumptions made by the dichromatic reflection model are:

1. There is a single light source that can be a point source or an area source;

2. The illumination has a constant PSD across the scene;

3. The amount of illumination can vary across the scene.

For what concerns the surface properties, the model assumes that:

1. The surface is opaque;

2. The surface is not optically active (no fluorescence);

3. The colourant is uniformly distributed

The assumption of illumination being due to only one source of illumination is limiting the application of such models to scenes where there is a dominant illuminant. The assumptions about the surface are typical for reflection models and not too unrealistic.

\section{Illuminant estimation}

The aim of the illuminant estimation is to imitate the human ability to separate the illumination PSD from the surface reflectance. Several approaches have been proposed; this includes methods relying on linear models (Ho et al., 1990), neural networks (Barnard et al., 2002), reliance on highlights and mutual reflection (Klinker et al., 1990), and Bayesian and probabilistic approaches (Sapiro, 1999).

McCamy et al. (McCamy and Calvin, 1992) derived a simple equation to compute correlated colour temperature from CIE 1931 chromaticity coordinates $x$ and $y$, which is useful in designing sources to simulate CIE illuminants.

\section{Vision system}

The light emitted by sources of illumination and modulated by surfaces in the scene arrives at the capturing sensors of the colour vision system that is observing the scene. The vision system senses the captured electromagnetic signal and then transforms the information carried by light into a colour image of the physical world.

Colour Charge Coupled Device (CCD) camera, which is an example of a typical vision system, contains a set of sensors that convert electromagnetic energy into electric signals, which are, then sampled and quantized. Conventional CCD cameras insert colour filters, with different spectral sensitivity to the various wavelengths, over each sensory element, typically red, green, and blue filters to obtain colour information. Figure 3 shows the spectral sensitivities of the Sony ICX098BQ (Sony ICX098BQ) CCD sensors, excluding lens characteristics and light source characteristics, as an example of a typical surveillance camera. Apart from the spectral sensitivity of the colour filters, the formation of digital image colour values includes other factors, such as lens characteristics, and the electronics of the camera.

Once a material surface is hit by a bundle of light emitted by an illuminant, the electromagnetic waves may be transmitted, absorbed, or reflected back into the air. The quantities of transmitted absorbed and reflected energy sum to the incident energy at each wavelength. Those quantities are typically measured in relative terms as a fraction of the incident energy. Some materials may emit light, or fluorescence effect may occur, where the material absorbs light at specific wavelengths and then reflect light at different wavelengths. The surface's absorption, transmittance and reflectance are obtained. In this report, materials are assumed to be opaque, so light transmission through the material is not considered. The materials are assumed not to be fluorescence, and so the emission is not considered to focus on reflection.

The surface spectral reflectance of a material refers to the ability of the material to reflect different spectral distributions when some light shines on it. A reflectance model is a function that describes the relationship between incident illumination PSD and reflected light at a given point on a surface and at each wavelength. $S(\lambda)$ is defined as the ratio between the reflected PSD to the incident PSD. Different materials have different mechanisms of reflection, optically; most materials can be divided into two categories:

1. Homogeneous materials and,

2. Inhomogeneous materials

Homogeneous materials have a uniform refractive index throughout their surface and bodies.

On the other hand, inhomogeneous materials have varying refractive index throughout their surfaces and bodies. If a light hits its surface, part of the light reflects (specular reflection), while the other part enters the object and then reflects back to the air causing diffuse reflection. Such material's surfaces are known as diffuse (matte) surfaces or Lambertian surfaces.

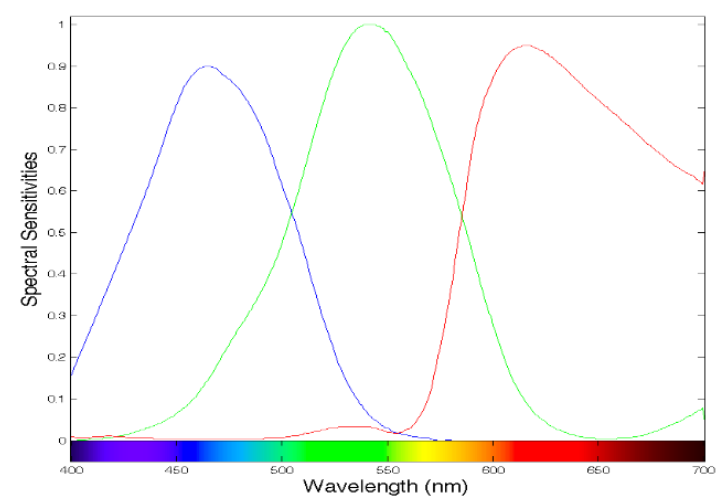

Figure 3: Spectral sensitivity characteristics of the Sony ICX098BQ camera. 


\section{COMPUTATIONAL MODEL}

Our method can be described in eight stages. The first stage estimates the SSR based on the dichromatic colour reflectance model. This approach uses image formation models to computationally estimate, from the camera output, a consistent physics-based color descriptor of the spectral reflectance of surfaces visible in the image, and then to convert the fullspectrum reflectance to Long $(L)$, Medium $(M)$, Short $(S)$ Cone responses and Rod $(R)$ response. This method represents a new approach to image enhancement, using explicit hypotheses about the physics that create images. This step makes our proposed different from the original model, (Pattanaik et al., 1998), in that it relies on models, which can represent wide classes of surface materials. It makes use of the pre-trained linear SSR models, shown in Figure 2, to represent the SSR of the objects in the scene. For what concerns the surface properties, this model assumes that: the surface is opaque; not optically active (no fluorescence) and the colourant is uniformly distributed.

The cones and rod spectral characteristics shown in Figure 4 are multiplied by the SSR and the result is integrated to obtain the cone and rod responses, four images representing the calibrated photoreceptor responses $(L, M, S, R)$, where they represent, Long, Medium, Short and rod signals respectively. Those four signals will be subjected to spatial processing.

The second stage, is the spatial decomposition of these four images, by applying the Laplacian pyramid (difference-of-Gaussian pyramid) approach (Burt and Adelson, 1983). We generate seven Gaussian pyramid's levels. Each level of the Gaussian pyramid represents a low-pass image limited to spatial frequencies half of those of the next higher level.

The third stage is the gain control stage, where the difference-of-Gaussian images are then converted to adapted contrast signals using a luminance gain control. The gains are set using TVI-like functions that represent the increment thresholds of the rod and cone systems and the growth in response required to allow perceived contrast to increase with luminance level (sub Weber's law behaviour). Performing the gain control at this point in the model allows proper prediction of chromatic adaptation effects. T

The fourth stage, is the opponent colour processing, where the model uses the transform of (Hunt, 1995) to transform the adapted contrast images for the cones into opponent signals, that will result in the transformation of the $L, M, S$-cone signals to $A$, $C_{1}, C_{2}$, that represent luminance, red-green, and yellow-blue opponent signals respectively.

The fifth stage is the adapted contrast transducers, where the adapted contrast signals are then passed through contrast transducer functions (Watson and Solomon, 1997). Different transducer functions are applied to each spatial frequency mechanism to model psychophysically derived human spatial contrast sensitivity functions. One of the key functions of the transducers is to set the threshold level (200) such that image content that is imperceptible for a given set of viewing conditions can be removed.

The sixth stage is the combination of rod and cone signals, in previous stages it is important to keep the rod signals separate from the cones to appropriately integrate their unique adaptation and spatial vision properties. After the contrast transducers, the rod and cone signals can be combined to produce signals that represent the three-dimensional colour appearances of the input image. At this stage, the model has three channels representing achromatic, red-green, and yellow-blue apparent contrast for 6 band-pass mechanisms.

The seventh stage is the treatment of the low-pass image, where the lowest level ( $7^{\text {th }}$ level) low-pass image from the up-sampled Gaussian pyramid must be retained in order to reconstruct an image from the adapted contrast images that have been passed through the model. The approach used, produces maximum dynamic range compression, by multiplying each pixel in the low-pass image by a gain factor derived from the pixel value itself. This treatment mimics the visual response assuming that the observer fixated on each and every image location and judged them completely independent of one another.

The eighth and last stage, is the image reconstruction stage, where the final outputs of the model are, signals in an achromatic and two chromatic channels, six spatial band-pass mechanisms and a low-pass image. In the last stage, the model must be inverted, this procedure does not "undo" the processes of the model since the threshold and saturated ion procedures are accomplished.

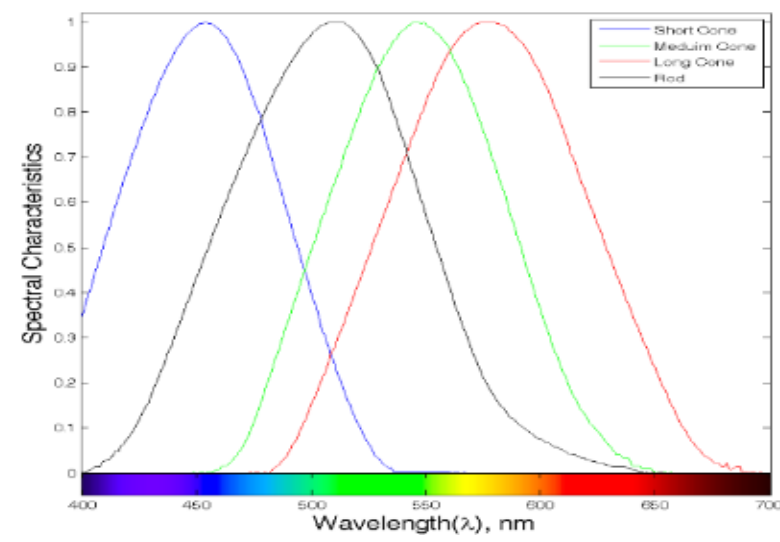

Figure 4: Cones and Rod spectral characteristics 

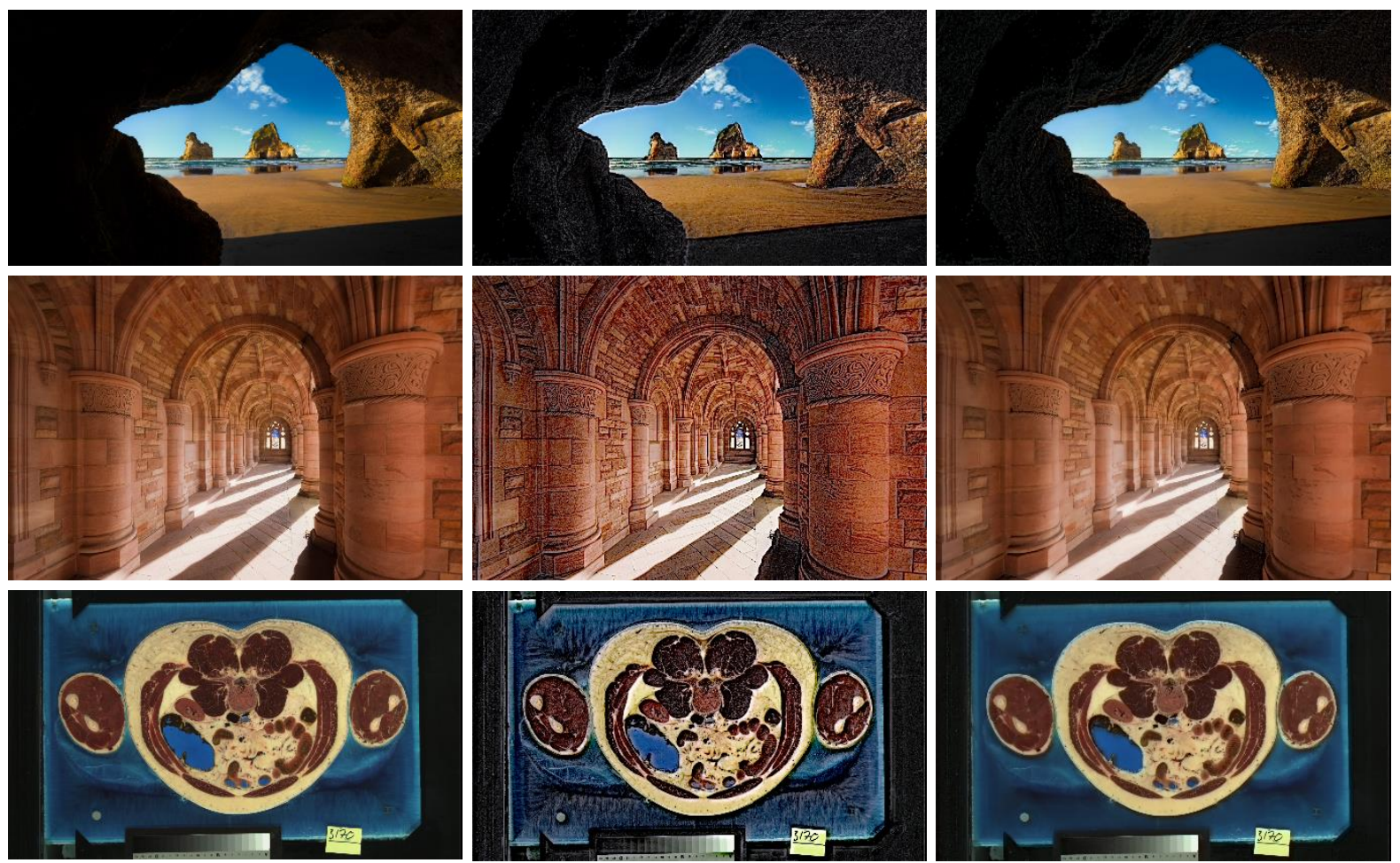

Figure 5: Illustrative visual comparison of the results obtained, the original image (left), the output of the multiscale model of adaptation (middle) and physics-based retina-inspired model (right).

\section{ILLUSTRATIVE VISUAL COMPARISON}

We have implemented our computational model using Matlab. The results of our implementation, are evaluated by visually comparing the results of the multiscale model of adaptation method (Pattanaik et al., 1998) and our proposed model.

The subjective assessment method was selected to initially evaluate results obtained by the proposed physics-based retina-inspired model. This would allow the authors to identify potential application where image/video processing algorithms could be improved by applying the proposed model.

The first image in the top section of Figure 5 shows a view taken from a sea cave looking across a beach. In this image there is a shadow in the foreground at the entrance to the cave. The wall of the cave appears to be saturated almost black, and the surface details are not defined. The middle image in the top section is the output obtained by the application of the multiscale model of adaptation. The result shows that the model concentrated on predominantly the darker areas highlighting, in particular, the shadowed areas. This resulted in an almost CGI rendition of the original image and appeared unrealistic. When applying our proposed model (right-hand image top section of Figure 5 however, the definition appears to be more precise and clearer. On the other hand, the visual model used in the middle image highlights the details of the sand patterns within the cave shadow.

The first image of the second row (from the lefthand side) is of a cloister in a monastic building highlighting the shadows bouncing off sandstone columns. The image also defines the details in the carvings above the pillars. The second image of the same row is less natural due to oversaturation of detail. However, the intricate carvings are clearly highlighted but to some extent exaggerated. The third image in the same row on this occasion is visually very similar to the first image.

The bottom left image in Figure 5 shows an example of a medical image. The output of the multiscale adaptation model is showing good improvement by extracting details such as blood vessels that could improve medical diagnosis. Although, the results obtained by using our model (right bottom of Figure 5 shows fewer details, it could improve the segmentation quality of certain objects, depending on the required application. 

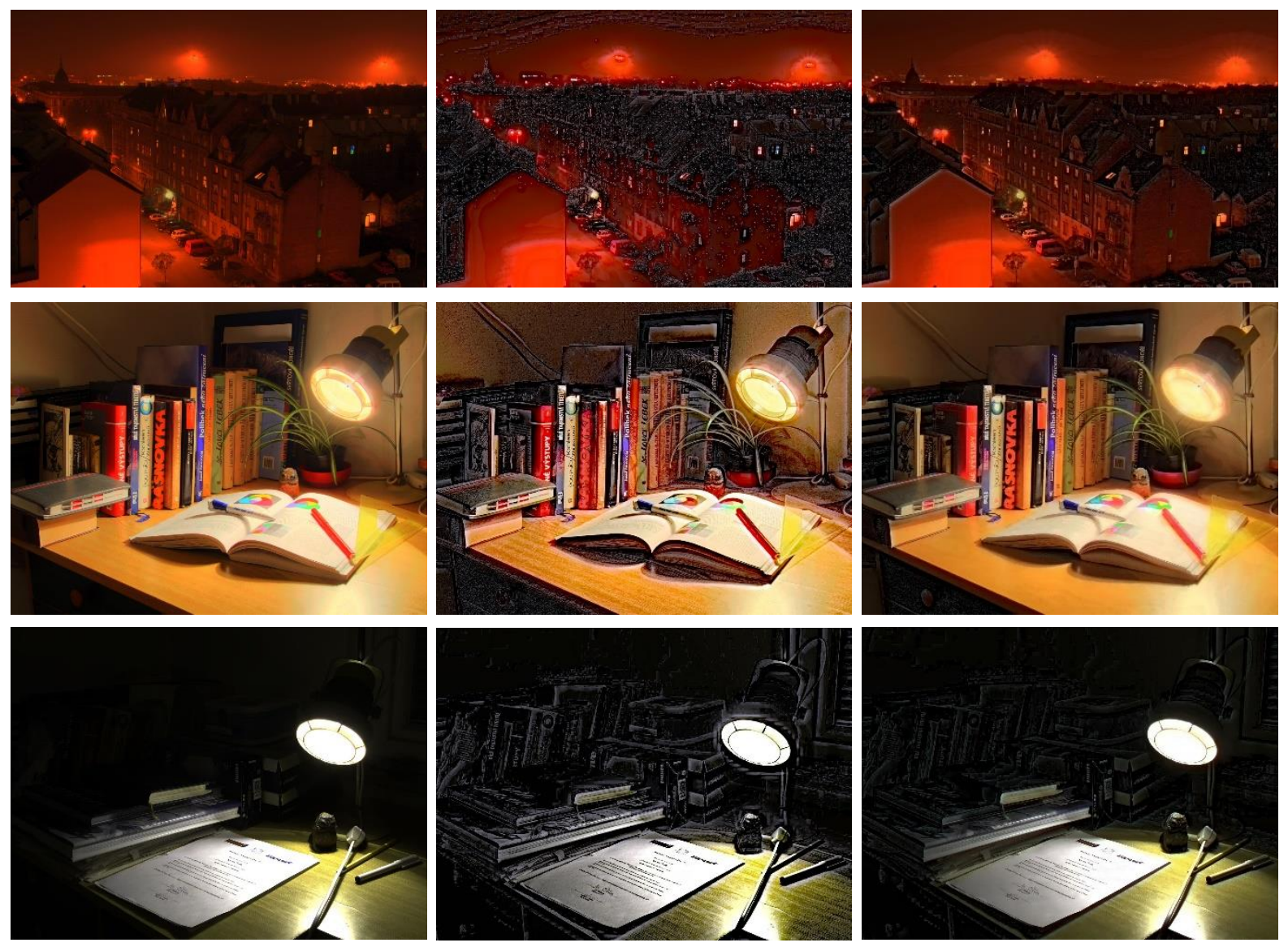

Figure 6: Illustrative visual comparison of the results obtained, the original image (left), the output of the multiscale model of adaptation (middle) and physics-based retina-inspired model (right).

The image on the top of Figure 6 shows an image taken at night. The gable ends, roofs and building facades are blurred. The results obtained by the application of multiscale adaptation model (the top middle image of Figure 6) shows that too many artefacts were identified, make it difficult to identify important features such as roof tops or eaves. In comparison, the top left image of Figure 6 shows the output of our proposed model. This brings out the finer details with a reasonable level of unwanted artefacts. Clear lines of the gable end, roofs and building facades can be also observed.

The middle left image of Figure 6 represents a bright image with glare from objects. The multiscale adaptation model (the second image in the middle row) although finds more features, it also detects unwanted artefacts on a desk draw, and this can give misleading results. It can be also noticed that some book titles are distorted due to unwanted artefacts. With the proposed model (middle right of Figure 6) the output of our algorithm detects less unwanted data, yet still provides good sharpness of objects.
Finally, the bottom left the image in Figure 6 shows similar scenario as the middle image i.e. a desk with a lamp and books on one side of the table. However, this a darker image where the lamp was the only source of light, and, therefore, the book titles are not visible. Both models improved the image and clear book edges are visible, and book titles can be legible. However, the multiscale adaptation model (the second image in the last row of Figure 6) generates unwanted mist effect around the table lamp.

The computational complexity for the proposed algorithms is evaluated regarding average processing time for one frame. The presented results are obtained using a personal computer (processor: Intel i7 $3 \mathrm{GHz}$, Memory: 16GB RAM), an implementation in Matlab (version: 8.3, release: R2014a). The multiscale model of adaptation method requires $0.44 \mathrm{sec}$ to process a 240x320 image while our proposed algorithm requires $1.08 \mathrm{sec}$. 


\section{CONCLUSION}

In this paper, we have developed a novel physicsbased and retina-inspired image/video enhancement technique that integrates a physics-based image formation model, the dichromatic model, with a retina-inspired computational model, a multiscale model for adaptation.

We have embedded both contrast and colour constancy by extracting physical features from the camera output; this approach is unlike other image enhancement algorithms that use the camera output directly without considering its physical meaning.

The estimation of the spectral characteristics of the dominant illuminant allows the proposed technique to adapt itself to different illumination conditions; this means that it would be applicable for a variety of scenes and not to be limited to certain environments.

Our results have shown that the estimation and use of physics-based spectral image representations, deduced from the dichromatic model, represent a more realistic input to the retina-inspired models and would mimic the signal received by the human eye.

\section{REFERENCES}

Bajcsy R., Lee S., \& Leonardis A., (1990). Colour image segmentation with detection of highlights and local illumination induced by inter-reflections In Proceedings of ICPR, pp. 785-790.

Barnard, K., Cardei, V., \& Funt, B. (2002). A comparison of computational color constancy algorithms. I: Methodology and experiments with synthesized data. Image Processing, IEEE Transactions on, 11(9), 972984.

Berns, R. S., Motta, R. J., \& Gorzynski, M. E. (1993). CRT colorimetry. Part I: Theory and practice. Color Research \& Application, 18(5), 299-314.Burt, P.J., and Adelson, E.H. (1983) The Laplacian Pyramid as a Compact Image Code. IEEE Transaction on Communication, 31(4), 532-540.

Ciurea, F., \& Funt, B. (2004). Tuning retinex parameters. Journal of Electronic Imaging, 13(1), 58-64.

Finlayson, G. D. (1995). Color constancy in diagonal chromaticity space. In Computer Vision, 1995. Proceedings, Fifth International Conference on (pp. 218223). IEEE.

Frankle, J. and McCann, J., (1983). Method and apparatus for lightness imaging, US Patent, May 1983, number Patent 4384336.

Georgeson, M. A., and Sullivan, G. D. (1975). Contrast constancy: deblurring in human vision by spatial frequency channels. The Journal of Physiology, 252(3),

Ho, J., Funt, V., \& Drew, M. S. (1990). Separating a color signal into illumination and surface reflectance components: Theory and applications. Pattern Analysis and Machine Intelligence, IEEE Transactions on, 12(10), 966-977.

Hunt, R.W.G. (1995). The Reproduction of Color. 5th edition, Kingstonupon-Thames, England: Fountain Press

Klinker, G. J., Shafer, S. A., \& Kanade, T. (1990). A physical approach to color image understanding. International Journal of Computer Vision, 4(1), 7-38.

Land, E. H. (1986). Recent advances in Retinex theory. Vision research, 26(1), 7-21.

Land, E. H., \& McCann, J. (1971). Lightness and retinex theory. JOSA, 61(1), 1-11.

Maini, R., \& Aggarwal, H. (2010). A comprehensive review of image enhancement techniques. arXiv preprint arXiv:1003.4053.

Maloney L. T., (1986). Evaluation of linear models of surface spectral reflectance with small numbers of parameters. In: Journal of the Optical Society of America A, vol. 3, pp. 1673-1683.

Marimont D. H., and Wandell B. A., (1992). Linear models of surface and illuminant spectra. In: Journal of the Optical Society of America A, vol. 3, pp. 1673-1683.

Marr, D., \& Vision, A. (1982). A computational investigation into the human representation and processing of visual information. WH San Francisco: Freeman and Company.

McCamy and Calvin S., (1992). Correlated colour temperature as an explicit function of chromaticity coordinates. In: Journal of Colour Research \& Application, vol. 17, no. 2, pp. 142-144.

Morel, J. M., Petro, A. B., \& Sbert, C. (2010). A PDE formalization of retinex theory. Image Processing, IEEE Transactions on, 19(11), 2825-2837.

Parkkinen, J. P., Hallikainen, J., \& Jaaskelainen, T. (1989). Characteristic spectra of Munsell colors. JOSA A, 6(2), 318-322.

Pattanaik, S. N., Ferwerda, J. A., Fairchild, M. D., \& Greenberg, D. P. (1998, July). A multiscale model of adaptation and spatial vision for realistic image display. In Proceedings of the 25th annual conference on Computer graphics and interactive techniques (pp. 287298). ACM.

Reddy, A. A., Jois, P. R., Deekshitha, J., Namratha, S., \& Hegde, R. (2013). Comparison of image enhancement techniques using retinex models 1, 1-6.

Saichandana, B., Ramesh, S., Srinivas, K., \& Kirankumar, R. (2014). Image Fusion Technique for Remote Sensing Image Enhancement. In ICT and Critical Infrastructure:

Sapiro G., (1999). Colour and illuminant voting. In: IEEE Transaction on Pattern Analysis and Machine Intelligence, vol. 21, no. 11, pp. 1210-1215.

Sedky, M., Moniri, M., \& Chibelushi, C. C. (2014). Spectral-360: A Physics-Based Technique for Change Detection. In Computer Vision and Pattern Recognition Workshops (CVPRW), 2014 IEEE Conference on (pp. 405-408). IEEE.

Watson, A.B. and Solomon, J.A. (1997) Model of Visual Contrast Gain Control and Pattern Masking. J. Opt. Soc. Am. A, 14(9), 2379-2391. 